\title{
Intraluminal Application of Vascular Endothelial Growth Factor Enhances Healing of Microvascular Anastomosis in a Rat Model
}

\author{
Manfred Infanger ${ }^{a} \quad$ Mehdi Shakibaei $^{b, d}$ Peter Kossmehl ${ }^{c}$ Siri M. Hollenberg ${ }^{a}$ \\ Jirka Grosse $^{\mathrm{a}}$ Shideh Faramarzi ${ }^{\mathrm{c}}$ Gundula Schulze-Tanzil ${ }^{\mathrm{b}}$ Martin Paul $^{\mathrm{c}}$ \\ Daniela Grimm
}

${ }^{a}$ Department of Trauma and Reconstructive Surgery, ${ }^{b}$ Institute of Anatomy and ${ }^{\mathrm{c}}$ Institute of Clinical Pharmacology and Toxicology, Charité - University Medicine Berlin, Berlin, and ${ }^{\mathrm{d} I n s t i t u t e}$ of Anatomy, Ludwig Maximilians University, Munich, Germany

\section{Key Words}

Vascular endothelial growth factor - Anastomosis •

Endothelium · Collagen · Extracellular matrix

\begin{abstract}
Background: Early reconstitution after injury to the endothelium is an important feature for reducing a number of vessel wall pathologies. We investigated the effect of vascular endothelial growth factor (VEGF) and its impact on the vascular remodeling process and reendothelialization after microsurgery. Methods and Results: Microvascular anastomosis was performed in the rat femoral artery. One group was treated with intraluminal administration of VEGF and the other with vehicle. We investigated morphological, ultrastructural and immunohistochemical changes of the vascular wall and the reendothelialization process. After 10 days, reendothelialization was significantly faster in VEGF-treated rats. Transmission electron microscopy revealed a complete healing in contrast to vehicle-treated vessels. Moreover, extracellular matrix proteins, such as fibronectin, collagen types I, III and IV, were significantly increased. Furthermore, VEGF treatment significantly induced VEGF receptor 2, flk-1, osteopontin and TGF- $\beta_{1}$ proteins. Conclusions: Our data clearly document for the first time that
\end{abstract}

intraluminal treatment with VEGF is beneficial to the healing process in vascular microsurgery. Osteopontin and TGF- $\beta_{1}$, both induced by VEGF, may play an important role in the vascular remodeling process. Our results provide clear evidence that VEGF application may represent a useful strategy in accelerating reendothelialization and improving vascular healing after microsurgery.

Copyright $(2005$ S. Karger AG, Basel

\section{Introduction}

The loss of endothelium as occurs after vascular trauma, microvascular transplantation, and replantation in reconstructive surgery is associated with increased thrombosis, smooth muscle cell (SMC) proliferation and neointimal formation, processes that often lead to stenosis and vessel occlusion. It is clinically important to achieve fast and perfect regeneration of the endothelial cell layer after microvascular anastomosis to increase the survival of replanted or transplantated tissue.

The kinetics of reendothelialization (rET) after microanastomosis of vessels are not completely understood. Vascular endothelial growth factor (VEGF) is one of the most potent mediators of vascular regulation in angiogen-

\section{KARGER}

Fax +4161306 1234 E-Mail karger@karger.ch www.karger.com (c) 2005 S. Karger AG, Basel $1018-1172 / 05 / 0423-0202 \$ 22.00 / 0$

Accessible online at: www.karger.com/jvr
Dr. Manfred Infanger

Head of Reconstructive Surgery, Department of Trauma and Reconstructive Surgery

Charité, University Medicine Berlin, Benjamin Franklin Campus

Hindenburgdamm 30, DE-12200 Berlin (Germany)

Tel, +49 308445 4850, Fax +49308445 1762, E-Mail manfred.infanger@charite.de 
esis and vascular permeability [1-4]. VEGF, also called 'vascular permeability factor', is an endothelial cell-specific mitogen secreted as a $45-\mathrm{kDa}$ protein that consists of two subunits which does not induce cell proliferation of other cell types. There exist five human isoforms with different numbers of amino acids (VEGF 121, 145, 165, 189, 206), generated by alternative splicing of the mRNA from a single gene comprising eight exons [2]. VEGF 121 and VEGF 165 are the only soluble isoforms and are the most abundant ones. VEGF 165 is the most powerful stimulator of endothelial cell proliferation [5]. VEGF 121 is responsible for the increase in vascular permeability [6]. To date, five different members of the VEGF family have been discovered: VEGF-A (165), VEGF-B, VEGFC, VEGF-D and VEGF-E. Only VEGF-A, VEGF-B, and VEGF-E modulate vascular angiogenesis and permeability. VEGF-C and VEGF-D modulate lymphatic angiogenesis. In addition to its mitogenic effect, VEGF has been shown to increase the permeability of the peripheral circulation and endothelial cell monolayers $[5,6]$. The major factor seems to be VEGF, referred to also as VEGF-A. The expression of VEGF is controlled by differentiation, transformation, and oxygen supply. The effect of VEGF is regulated by two receptor tyrosine kinases, VEGFR-1/flt-1 and VEGFR-2/flk-1/KDR, which are almost exclusively located on endothelial cells.

The aim of this study was (1) to measure if local VEGF application can improve rET after microanastomosis and (2) to investigate the impact of VEGF on the vascular remodeling process in vessels with or without VEGF treatment.

\section{Materials and Methods}

\section{Animal Grouping}

Adult male Sprague-Dawley rats (body weight 250-300 g) were obtained from Charles River Wiga (Sulzfeld, Germany). They were maintained on standard rat chow (H1003, Alma KG, Kempten, Germany) with water ad libitum. All animals were individually housed in a 12-hour dark/light cycle-controlled room. The protocols were approved by the local standing committee on animal research. The investigation conforms with the 'Guide for the Care and Use of Laboratory Animals' published by the US National Institutes of Health (NIH Publication No. 85-23; revised 1985). A total of 40 rats were randomized to VEGF $(10 \mathrm{ng} / \mathrm{ml})$ [7] or vehicle (saline) treatment. The following groups were investigated: (1) day 0 : rats were sacrificed at $10 \mathrm{~min}$ after local VEGF or vehicle application ( $\mathrm{n}=5$, each group); (2) 10 rats were sacrificed at day 5 for histological examination ( $n=5$, each group), and (3) rats were sacrificed at day $10(\mathrm{n}=10$, each group). At each time point 2 vessels were collected (left femoral artery with anastomosis and right femoral artery without anastomosis).

VEGF Enhances Healing of Microvascular Anastomosis

\section{Surgical Procedure}

Rats were anesthetized by intraperitoneal application of thiopental sodium (100 mg/kg, i.p.) [8]. The skin over the femoral artery was shaved, swabbed with povidone-iodine solution, and incised. Using a surgical microscope (Zeiss, Oberkochen, Germany), microsurgical preparation of the nerve vessel sheath was performed. A segment of the left femoral artery was carefully dissected from surrounding tissues. A pair of microclamps (Acland, S + T Germany) was used. To interrupt blood flow, the vessel was sharply transected at the middle of the segment. After stripping of the adventitia and flushing of the lumen with heparinized saline $(1 / 10,000)$, a direct end-to-end repair was performed with Nylon 11/0 sutures (fig. $1 \mathrm{~A}$ ) with about 10 sutures (diameter of vessel: $0.6-1 \mathrm{~mm}$ ). After the anastomosis (fig. 1A-C) was performed, one group was treated with intraluminal (via anastomosis) application of recombinant rat VEGF 164 (Sigma, Deisenhofen, Germany) for $10 \mathrm{~min}(10 \mathrm{ng} / \mathrm{ml})$ by clamping. The other group was treated with vehicle for $10 \mathrm{~min}$ (saline). Subsequently, the clamp was removed. Perfusion was tested with two forceps by occluding the vessel distal to the anastomosis site with one pair of forceps. A short segment of the vessel distal to the first pair was emptied with a second pair of forceps closed, the proximal pair was released and it was noted whether and how fast the emptied segment of the vessel refilled. Immediate refilling indicated patency of the anastomosis site, slow refilling suggested vessel spasm, partial thrombosis, or technical error, and no refilling indicated thrombosis or severe technical error. After perfusion of the anastomosis, the skin was closed with $3 / 0$ Nylon sutures.

During surgery, a warming lamp was used to maintain the body temperature at $37^{\circ} \mathrm{C}$ until the animal recovered from anesthesia. All surgical procedures were performed by an experienced plastic microsurgeon.

\section{Follow-Up}

After 10 min or 5 or 10 days, the vehicle or VEGF-treated rats were sacrificed and the vessels (left femoral artery with anastomosis and right femoral artery without anastomosis) were excised for histological (fig. 1B, C), electron-microscopic, immunohistochemical, and Western blot analyses.

\section{Transmission Electron Microscopy}

Transmission electron microscopy followed routine protocols according to Shakibaei et al. [9]. After fixation and postfixation in $1 \%$ tannic acid $\left(0.1 \mathrm{M}\right.$ phosphate buffer) and $1 \% \mathrm{OsO}_{4}$ solution (0.1 $M$ phosphate buffer), the vessel was dehydrated in an ascending alcohol series. The samples were embedded in Epon and cut on a Reichert Ultracut (Reichert-Jung, Nussloch, Germany), which was followed by administration of $2 \%$ uranyl acetate/lead citrate for contrast. The vessels were examined using a transmission electron microscope (TEM 10, Zeiss).

\section{Histological Evaluation and Polarization Microscopy}

For histology, transverse tissue slices of one part of the vessels ( $\mathrm{n}=5$, each group, day 5 ) were cut at $2-3 \mathrm{~mm}$ away from the anastomosis and subjected to hematoxylin-eosin and Sirius red staining as a specific dye for connective tissue structures. The slices were immersed in Dubosq-Brasil solution, embedded in paraffin, and cut into 3- $\mu \mathrm{m}$ sections. Standardized staining was realized using a Robot-Stainer system (Robot-Stainer HMS 760, Microm GmbH). The amount of fibrosis was quantified after Sirius red staining by morphometry. Fibrosis was visualized using a Zeiss Axiophot micro- 

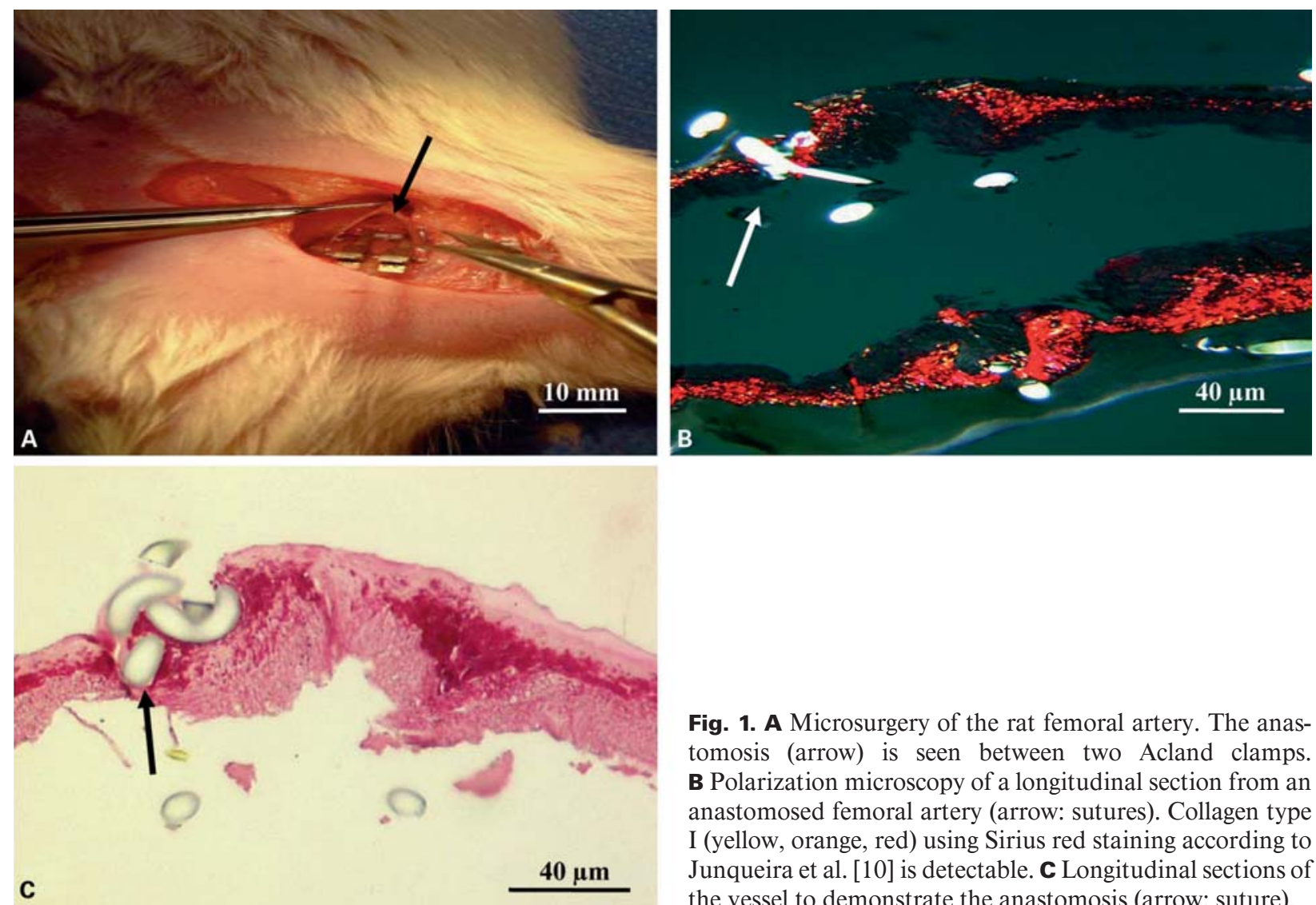

Fig. 1. A Microsurgery of the rat femoral artery. The anastomosis (arrow) is seen between two Acland clamps. B Polarization microscopy of a longitudinal section from an anastomosed femoral artery (arrow: sutures). Collagen type I (yellow, orange, red) using Sirius red staining according to Junqueira et al. [10] is detectable. C Longitudinal sections of the vessel to demonstrate the anastomosis (arrow: suture).

scope (Zeiss). Stenosis, thrombosis, rET, intimal hyperplasia, vascular structure, and aneurysm formation of the anastomosis site were documented. Polarization microscopy was performed according to Junqueira et al. [10]. Collagen type I presents a yellow, orange or red color, while collagen type III appears green.

\section{Immunohistochemical Staining}

The structural changes of the vessels at day 5 ( $n=5$, each group) were investigated using light microscopy (Olympus Optical, Hamburg, Germany). Frozen specimens were sectioned at $3 \mu \mathrm{m}$ and fixed with acetone $\left(-20^{\circ} \mathrm{C}\right)$ for $10 \mathrm{~min}$. Sections were selected to visualize antigen-antibody complexes using the indirect peroxidase technique. Incubation with the first antibody (osteopontin, MPIIIB10 ${ }_{1}$, University of Iowa, Hybridoma Bank, USA) was followed by incubation with a peroxidase-labeled second antibody. After repeated washing with phosphate-buffered saline, the slices were exposed to diaminobenzidine and $\mathrm{H}_{2} \mathrm{O}_{2}$, generating a brown color. Finally, the specimens were dehydrated and embedded with Entellan (Merck, Darmstadt, Germany). Controls for the antibody staining were negative.

\section{Quantitative Image Analysis}

The measurements of the arteries were performed using a video camera combined with a separated video control system (Sony MC3255, AVT-Horn $\mathrm{GmbH}$ ) adapted to a Zeiss Axiophot microscope.
Image analysis was performed with the use of freely available software (Scion Image 1.62a, Scion Co.) on a Power Macintosh $8200 / 120$ computer. The magnification was 10 -fold. Multiple sections $(\mathrm{n}=10)$ were examined for each specimen. The total, luminal, and medial vessel areas $\left(\mu \mathrm{m}^{2}\right)$ were measured. The media thickness was measured and the media-fibrosis-to-lumen ratio as well as media-to-lumen ratio were calculated.

For quantitative image analysis of osteopontin, the samples were digitalized, gray-scale images were transformed into binary images, and the relation of the osteopontin-immunostained area to the total luminal area of the vessel was determined.

\section{Western Blot Analysis}

Western blot analyses were performed at day 0 and 10 and carried out following routine protocols [11]. Antibodies against the following antigens were used for this study: VEGF-A, flk-1, TGF- $\beta_{1}$, collagen types I and IV (Santa Cruz, Santa Cruz, Calif., USA), fibronectin (Chemicon, Hofheim, Germany), collagen type III(Monosan, Beutelsbach, Germany), andosteopontin(MPIIIB10 ${ }_{1}$, University of Iowa, Hybridoma Bank, USA).

SDS-PAGE and immunoblotting were carried out following routine protocols [11]. The samples were homogenized by shearing forces in lysis buffer $(50 \mathrm{~m} M$ Tris- $\mathrm{HCl}, \mathrm{pH} 7.2 ; 150 \mathrm{mM} \mathrm{NaCl} ; 1 \%$ Triton X-100; $1 \mathrm{~m} M$ sodium orthovanadate; $50 \mathrm{~m} M$ sodium pyrophosphate; $100 \mathrm{~m} M$ sodium fluoride; $0.01 \%$ aprotinin; $4 \mu \mathrm{g} / \mathrm{ml}$ 

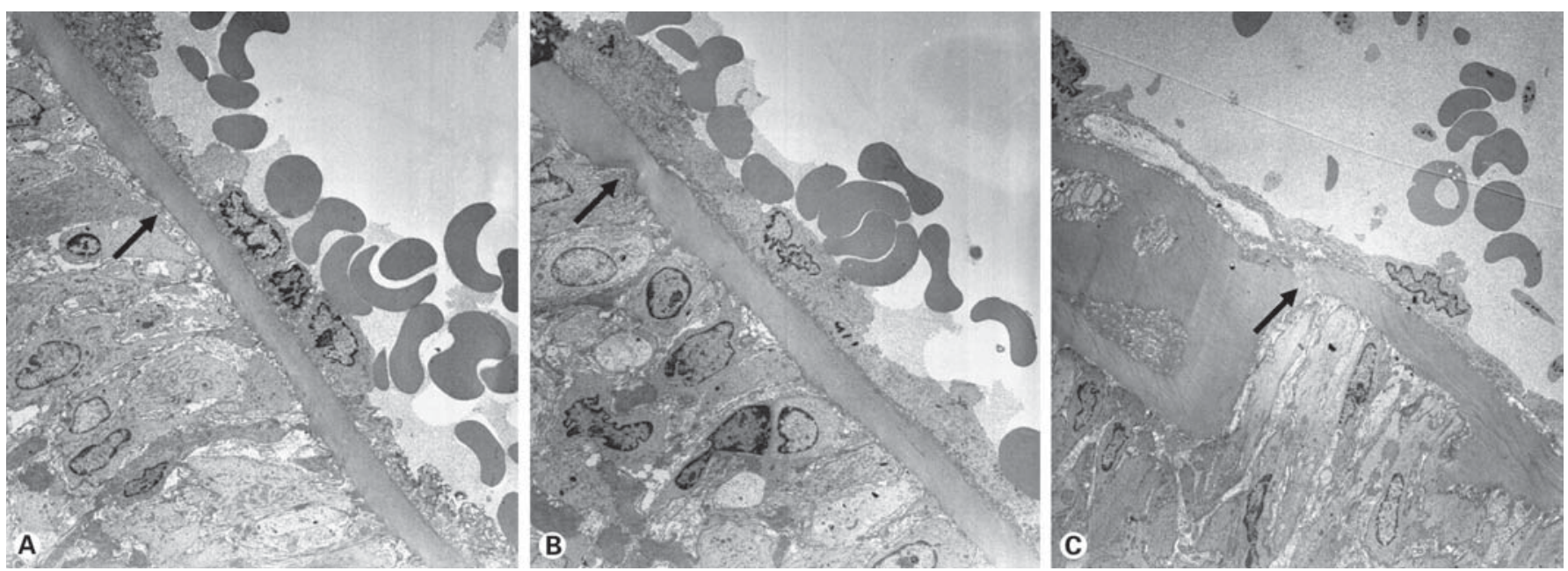

Fig. 2. Electron microscopy of the rat femoral artery. A Control, normal basement membrane (arrow) of a control microvessel. B Anastomosis of the microvessel with local administration of VEGF. After 10 days, the basement membrane defect is closed (arrow), healing is perceptible and rET is clearly detectable. C Anastomosis of the microvessel with vehicle administration. After 10 days, defect healing was noted, the basement membrane remained open (arrow). A-C Magnification $\times 4,000$.

pepstatin A; $10 \mu \mathrm{g} / \mathrm{ml}$ leupeptin, and $1 \mathrm{~m} M$ phenylmethylsulfonyl fluoride) on ice for $30 \mathrm{~min}$. For immunoblotting, equal amounts of total proteins $(50 \mu \mathrm{g}$ total protein, each fraction was loaded per lane) were separated on 7.5\% SDS-PAGE polyacrylamide gels under reducing conditions.

Subsequently, the homogenates were transferred onto a nitrocellulose membrane (Schleicher \& Schuell, Dassel, Germany) using a Transblot electrophoresis apparatus (Mini Trans Blot, Bio-Rad Laboratories, Richmond, Calif., USA) for $1 \mathrm{~h}$ at $120 \mathrm{~V}$. Membranes were blocked with 5\% (wt/vol) skim milk powder in PBS/0.1\% Tween 20 overnight at $4{ }^{\circ} \mathrm{C}$ and incubated with primary antibodies diluted in blocking buffer for $1 \mathrm{~h}$ at room temperature. After five washes in blocking buffer, membranes were incubated with alkaline phosphatase-conjugated secondary antibody diluted in blocking buffer for $30 \mathrm{~min}$ at room temperature. Membranes were finally washed 5 times in blocking buffer and 3 times in $0.1 M$ Tris ( $\mathrm{pH} 9.5$ ) containing $0.05 \mathrm{M} \mathrm{MgCl}_{2}$ and $0.1 \mathrm{M} \mathrm{NaCl}$; specific binding was detected using nitroblue tetrazolium and 5-bromo-4-chloro-3-indoyl-phosphate ( $p$ toluidine salt; Pierce, Rockford, Ill., USA) as substrates and was measured by densitometry (Personal Densitometer 50301, Molecular Dynamics, Krefeld, Germany). Protein determination was performed with the bicinchoninic acid system using BSA as a standard [11].

\section{Statistical Analysis}

Statistical analysis was performed using SPSS 10.0. Results are expressed as mean \pm SEM. Comparisons between multiple groups were assessed by one-way analysis of variance (ANOVA), including a modified least-significant difference (Bonferroni) multiple-range test to detect significant differences between two distinct groups, which were further analyzed using the Mann-Whitney U test. The strength of the relationship between two variables was assessed by calculation of the product-moment correlation coefficient (r). Statistical significance was accepted at the level of $\mathrm{p}<0.05$.

\section{Results}

\section{Patency Rate}

The forceps vessel flow test showed a 100\% patency rate in all surgery groups immediately after anastomosis. One vessel of the vehicle-treated animals was occluded by a thrombus.

\section{Effect of VEGF on Survival Rate}

All VEGF-treated animals with microsurgery survived the entire 10 days' observation period. In contrast, 2 rats of the vehicle-treated animals died. One animal died at day 2 because of hypovolemia due to bleeding because of a defect at the anastomosis, the other at day 8 because of pneumonia. Both deaths were not related to treatment.

\section{VEGF Enhances $r E T$}

Transmission electron microscopy revealed a complete rET and healing at day 10 postsurgery in VEGFtreated vessels (fig. 2B). Vehicle-treated vessels exhibited defective healing, as shown in figure $2 \mathrm{C}$.

\section{Investigation of Collagen and Osteopontin}

As osteopontin is involved in collagen organization, we analyzed the expression of collagen in vessels with and without anastomosis. Using Sirius red staining according to Junqueira et al. [10], a clear increase in collagen 

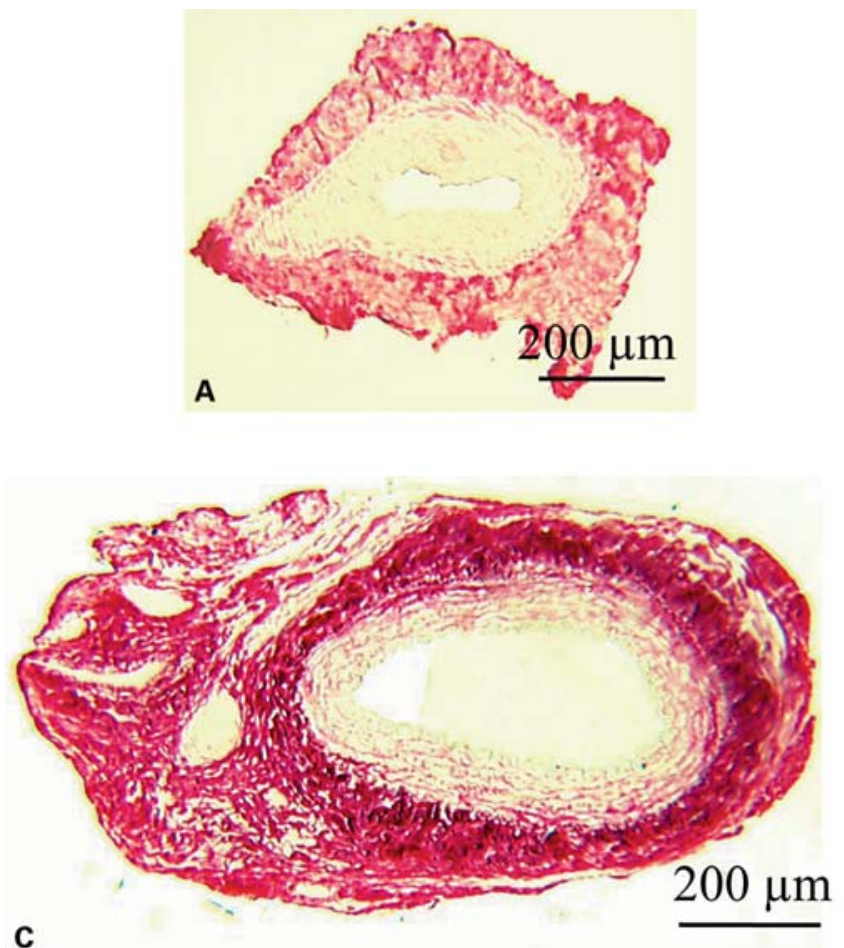

C
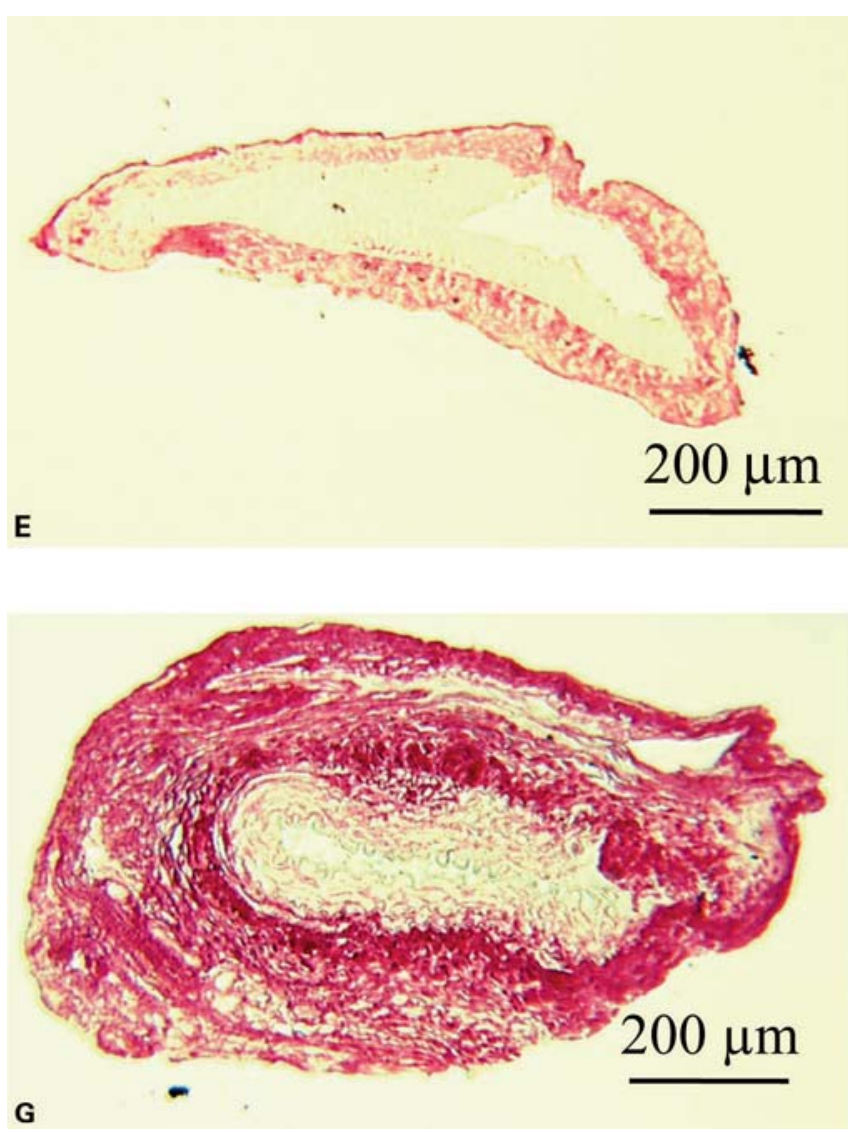
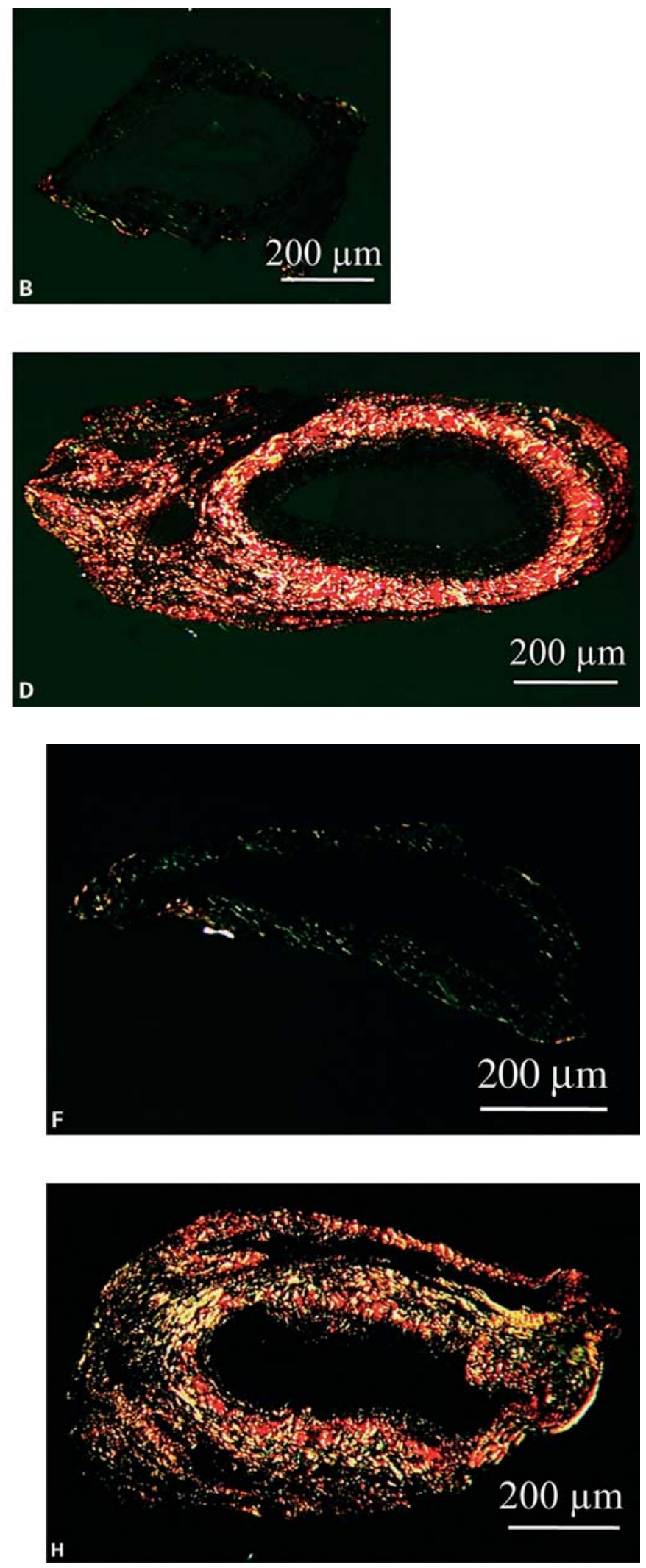

Infanger et al. 


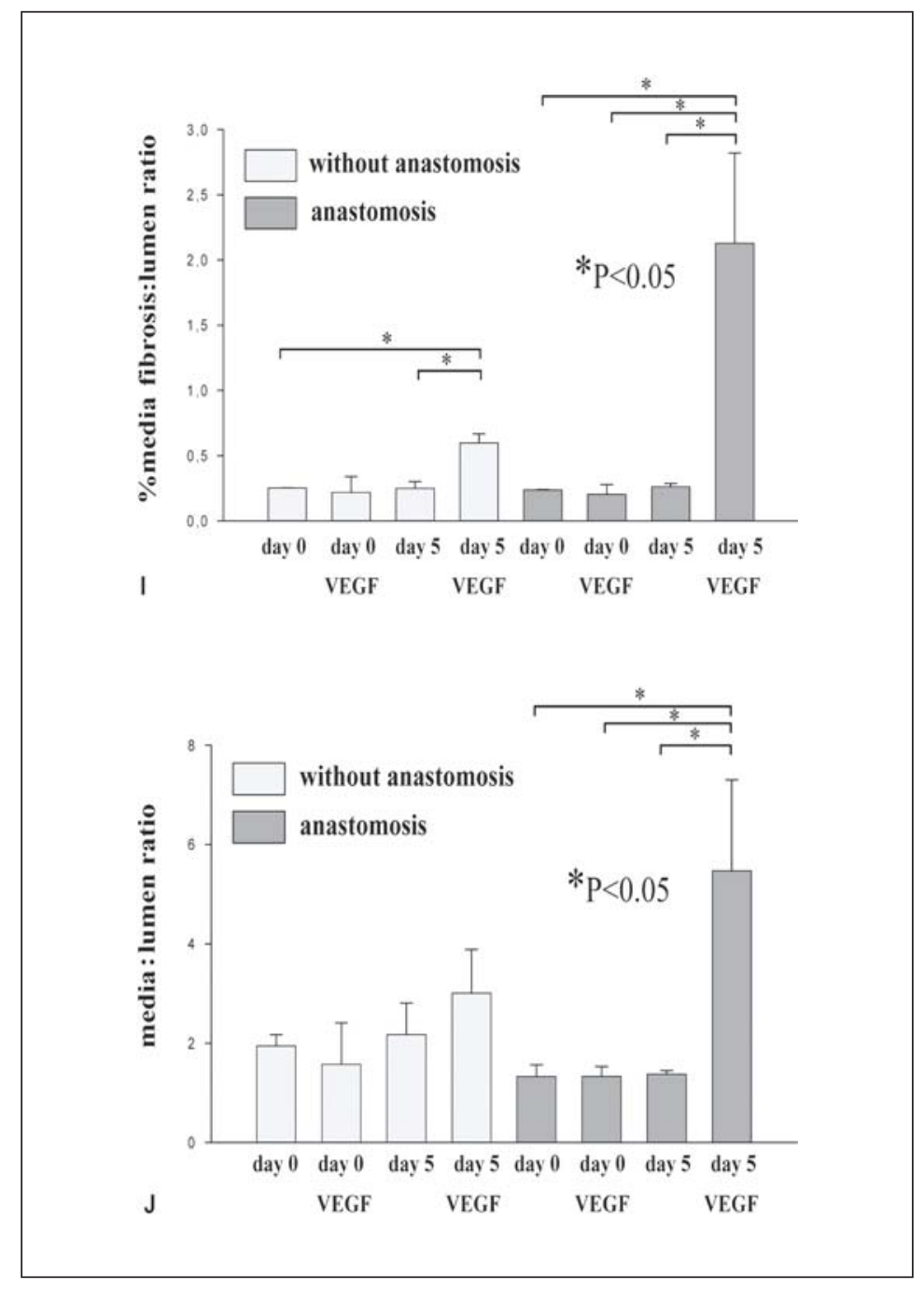

Fig. 3. Sirius red staining at day 5 postsurgery. A Control vessel of a vehicle-treated rat. B Polarization microscopy of the same section. Only little collagen type I (orange)- and III (green)-positive material is seen. C, D Vehicle-treated reanastomosed vessel with perivascular fibrosis. Polarization light revealed a clear accumulation of collagen type I (yellow, orange and red) as well as collagen type III (green). E, F Control vessel of a VEGF-treated animal. Polarization microscopic examination revealed a clear increase of collagen type III in the control vessel. G, H The anastomosed VEGF-treated vessel showed perivascular fibrosis and a striking accumulation of mainly collagen type III (green) compared to collagen type I (yellow, orange and red). I, J The data of quantitative image analysis are given. I At day 5 postsurgery, VEGF significantly increased the percentage of media fibrosis in vessels with and without anastomosis. J Media thickness is significantly increased in VEGF-treated vessels with anastomosis. type I was detectable in the perivascular space (fig. 3C, D) at day 5 postsurgery in vehicle-treated vessels with an anastomosis compared to the corresponding control group (fig. 3A, B). Intraluminal VEGF application induced an increase in collagen type III in the control vessel (fig. 3E, F) and an enormous accumulation in the vessels with anastomosis (fig. 3G, H). Collagen type I was also detectable in reanastomosed vessels (yellow, orange and red staining). The percentage of media fibrosis-to-lumen ratio is given in figure 3I. At day 5, VEGF significantly increased media fibrosis in vessels with and without anastomosis. The media-to-lumen ratio is clearly elevated in VEGF-treated vessels with anastomosis (fig. 3J).

Osteopontin was increased in the media in VEGFtreated vessels compared to the corresponding controls (fig. 4A-D). Quantitative image analysis revealed a clear increase of osteopontin in VEGF-treated vessels with and without anastomosis (fig. 4E).

\section{Effect of VEGF on VEGF-A and Flk-1 Protein}

Anastomosis. VEGF protein at day 0 was decreased as compared to the group without anastomosis. Restoration of VEGF expression at day 10 to control level is detectable. Application of VEGF induced VEGF-A protein immediately after surgery and it reached the highest level at 10 days after intervention (fig. 5A).

Flk-1 protein was reduced at day 0 in the anastomosed vessel and then clearly elevated at 10 days after surgery. VEGF induced the VEGF receptor after $10 \mathrm{~min}$ to the same level that was reached after 10 days with and without VEGF (fig. 5B). 

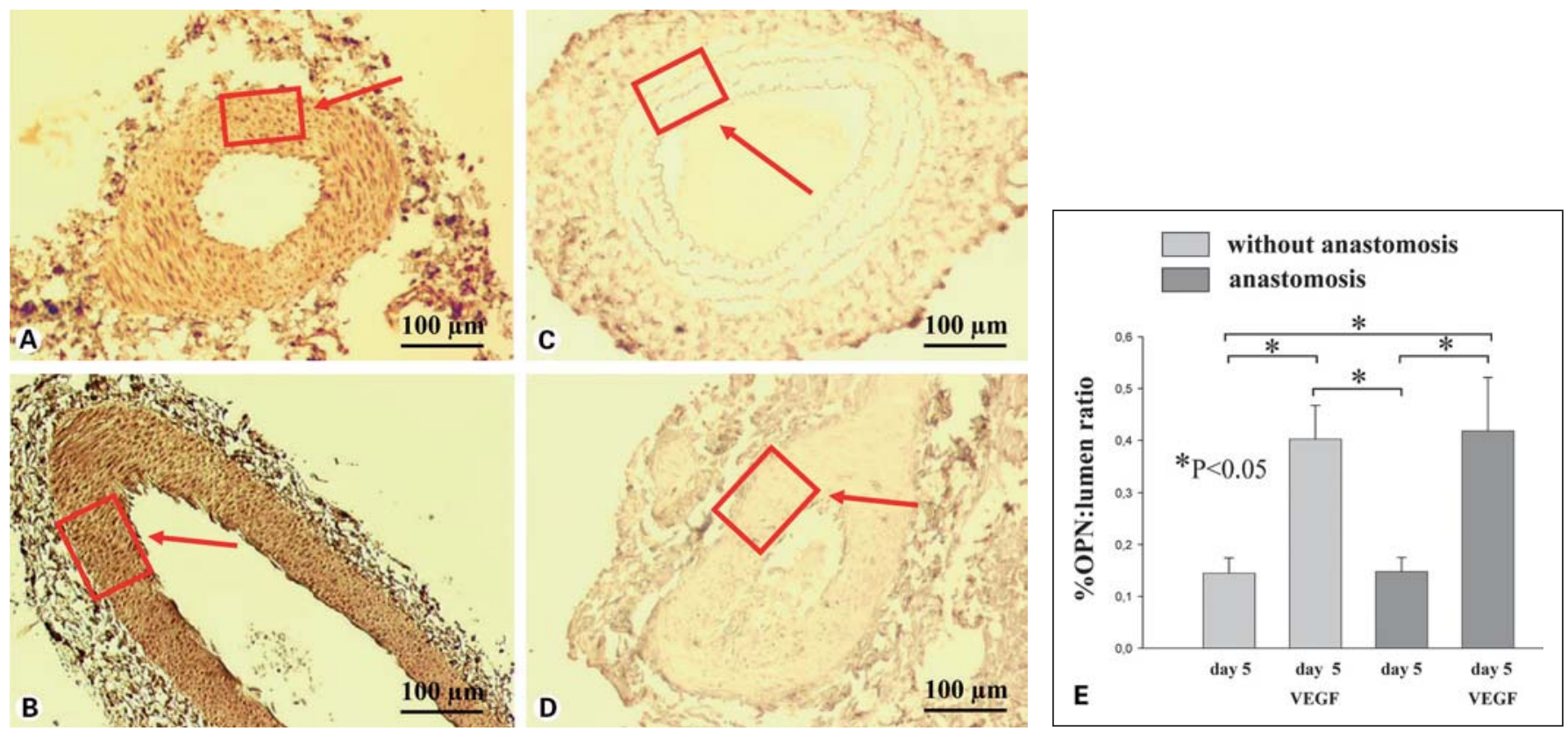

Fig. 4. Osteopontin staining, indirect immunoperoxidase method. A VEGF-treated control vessel. B VEGF-treated vessel with anastomosis. Osteopontin is detectable in the intima and media and mainly in the perivascular area of the femoral artery. C Vehicle-treated vessel. D Vehicle-treated vessel with perivascular fibrosis but with a lower amount of osteopontin at day 5 postsurgery. E Quantitative image analysis of osteopontin: osteopontin is significantly increased in all VEGF-treated vessels with and without anastomosis.

Control Vessel. VEGF protein content at days 0 and 10 with and without VEGF application was not significantly different. VEGF slightly increased the amount of VEGF protein at days 0 and 10 (fig. 5A). There was no change in flk-1 between the control and treated vessel (fig. $5 B)$.

\section{Impact of VEGF on the Extracellular Matrix}

Anastomosis. TGF- $\beta_{1}$ was increased at day 10 after surgery and this increase was more pronounced at day 10 in VEGF-treated rats. In addition, VEGF treatment resulted in immediate induction of TGF- $\beta_{1} 10 \mathrm{~min}$ after surgery (fig. 5C).

Control Vessel. VEGF significantly increased TGF- $\beta_{1}$ at day 0 and day 10 . TGF- $\beta_{1}$ on day 10 was similar to day 0 (fig. 5C).

Anastomosis. Osteopontin was significantly increased at 10 days after the surgery. VEGF treatment significantly enhanced osteopontin protein expression early after surgery and at day 10 (fig. 5D).

Control Vessel. Osteopontin was elevated in all VEGFtreated rats (fig. 5D).

Anastomosis. Collagen types I and III were clearly elevated at day 10 in VEGF-treated rats. At day 0, there was no significant difference compared to vehicle-treated vessels (fig. 6A, B). Collagen type IV was slightly elevated at day 10 in VEGF-treated animals (fig. 6C). VEGF significantly increased fibronectin at day 10 postsurgery. Moreover, there was a significant increase in fibronectin between day 0 and 10 (fig. 6D).

Control Vessel. Collagen types I and III were significantly increased at day 10 in VEGF-treated animals (fig. $6 \mathrm{~A}, \mathrm{~B})$. Collagen type IV was unchanged in all control groups (fig. 6C). On day 10, fibronectin was slightly, but not significantly elevated in vessels exposed to VEGF or vehicle (fig. 6D).

\section{Discussion}

VEGF, also known as vascular permeability factor, exerts its biological activity predominantly on endothelial cells. It has been shown to be an endothelial cell-specific mitogen in vitro and to be a key mediator of angiogenesis in vivo $[12,13]$. Moreover, our group has recently demonstrated that the VEGF serum level is correlated with tissue edema after burn injury [14]. Recent studies have shown that VEGF mRNA and protein are widely distrib- 


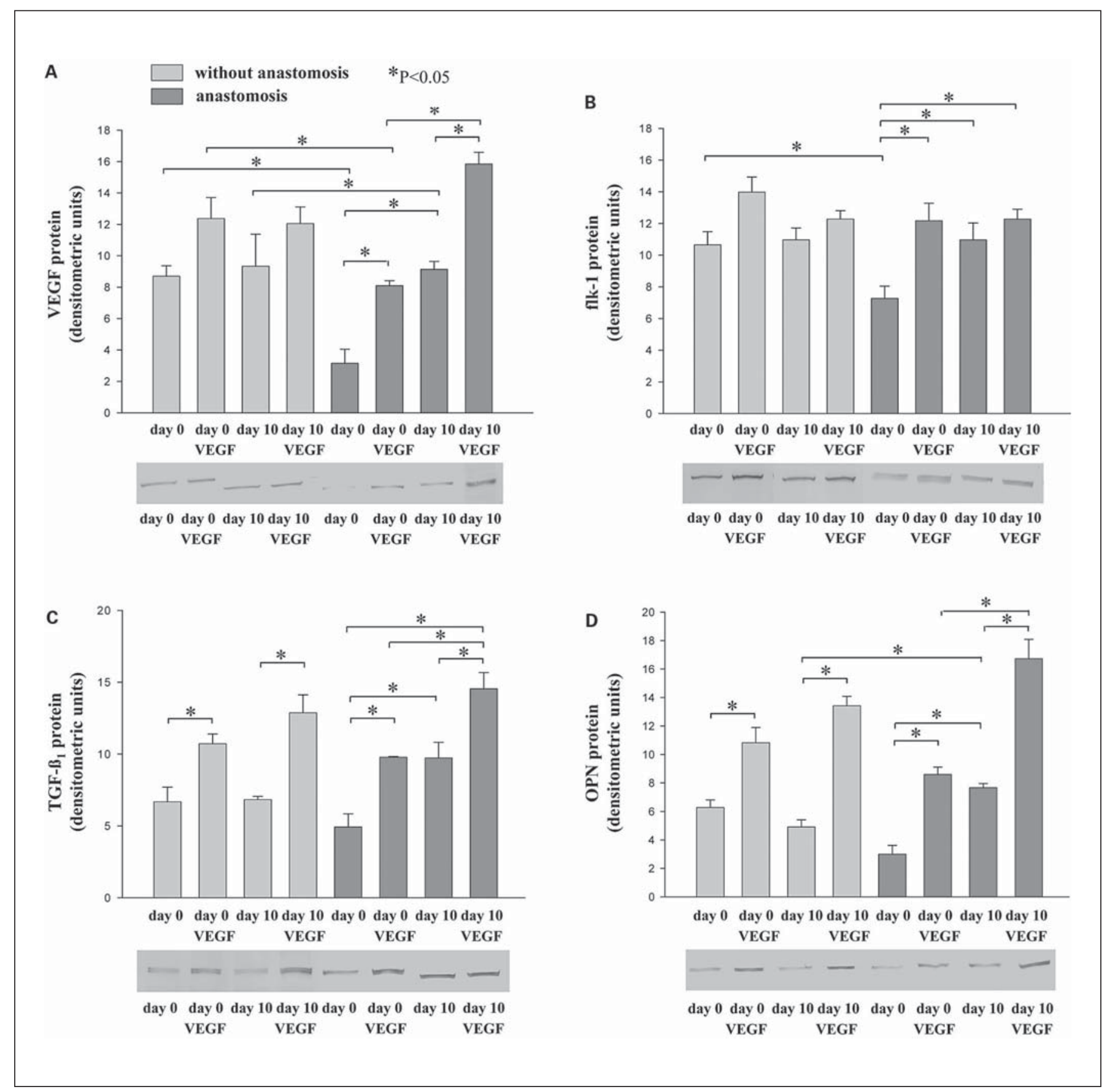

Fig. 5. Western blot analyses of VEGF (A) and the VEGF receptor 2 (flk-1) (B). The data for the control vessel (right femoral artery without anastomosis) and the left femoral artery with anastomosis are given. A The VEGF-treated microvessels exhibited a higher amount of VEGF protein as compared to the corresponding vehicle-treated groups. B VEGF receptor 2 (flk-1) is immediately upregulated after microsurgery in VEGF-treated vessels at day 0 . At day 10 postsurgery, flk-1 is increased in both anastomosed groups as compared to the vehicle-treated control group at day 0. Bars represent mean \pm SEM. Western blot analyses of TGF- $\beta_{1}(\mathbf{C})$ and osteopontin (D). The data for the control vessel (right femoral artery

without anastomosis) and the left femoral artery with anastomosis are given. C TGF- $\beta_{1}$ is early increased by topical VEGF administration in both the anastomosed vessel and the control vessel groups at days 0 and 10 . Moreover, TGF- $\beta_{1}$ protein is clearly elevated at day 10 in vehicle-treated vessels with anastomosis as compared to the day 0 group. D Osteopontin is early increased by topical VEGF administration in both the anastomosed vessel and the control vessel groups at days 0 and 10 . Moreover, osteopontin protein is clearly elevated at day 10 in vehicle-treated vessels with anastomosis as compared to the day 0 group. Bars represent mean \pm SEM. 


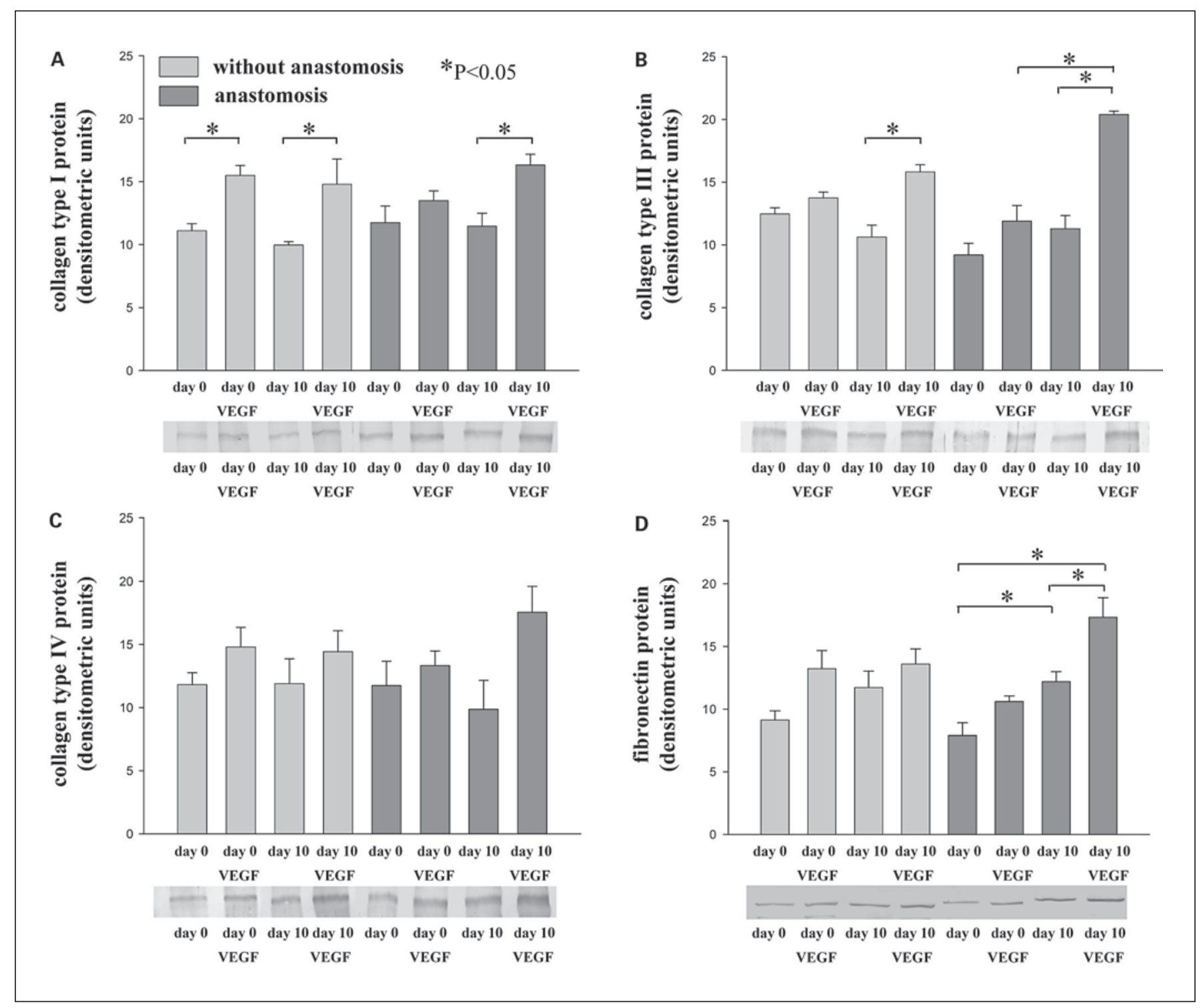

Fig. 6. Western blot analyses of collagen type I (A), collagen type III (B), collagen type IV (C) and fibronectin protein (D). The data for the control vessel (right femoral artery without anastomosis) and the left femoral artery with anastomosis are given. A Collagen type I is increased by VEGF application in vessels with and without anastomosis at day 10 postsurgery. B Collagen type III is clearly

uted in normal rat tissues and organs supporting the concept that VEGF may play an important role in the maintenance and control of vascular function $[15,16]$. It has been shown that in arterial reconstruction procedures using venous conduits, VEGF is significantly increased at $48 \mathrm{~h}$ in the vein graft and arterial anastomosis. VEGF expression in the vein graft normalizes within 4 weeks, but remains significantly elevated in the adjacent arterial elevated by VEGF administration at day 10 postsurgery. C Collagen type IV is slightly but not significantly increased at day 10 postsurgery. D Fibronectin protein is increased at day 10 compared to day 0 in vessels with anastomosis. In addition, topical VEGF application increased fibronectin at day 10 postsurgery. Bars represent mean \pm SEM. 
rial injury are poorly understood. Here, we demonstrate that VEGF is significantly induced in vascular anastomosis of rat femoral arteries 10 days postsurgery. Since VEGF-specific receptor flk-1 is expressed in rat femoral arteries and clearly upregulated at day 10 postintervention, we hypothesized that local application of VEGF after vascular injury would promote the vascular remodeling process in healing the wounded area. Moreover, we detected a decrease in VEGF/flk-1 protein expression at day 0 in vehicle-treated animals with anastomosis. This might be explained by the denudation of the vessel because endothelial cells express VEGF and flk-1. Restoration to control levels was achieved after healing at day 10 . In addition, at day 0 in VEGF-treated vessels with anastomosis, the amount of VEGF protein is comparable to the control vessel without surgery. This might be explained by an upregulation of VEGF protein in the remaining endothelial cells. In VEGF-treated rats, we detected complete healing 5 days postsurgery and the vascular remodeling process was completely finished in VEGF-treated animals. Previous investigations on the efficacy of VEGF to promote rET after arterial balloon angioplasty have been published $[18,19]$ reporting controversial data. Asahara et al. [18] showed that arterial gene transfer of phVEGF165 can accelerate rET at local and remote sites, leading to neointimal thickening and reduction in thrombogenicity. In contrast, Lindner and Reidy [19] reported on a lack of increased endothelial regrowth in response to VEGF. Moreover, in a second study they demonstrated that vascular SMCs express functional flt-1 receptors after arterial injury and that VEGF has synergistic effects with FGF-2 on SMC proliferation in vivo [20]. These effects are likely to be mediated by a VEGFmediated increase in permeability as well as a direct interaction between the VEGF and FGF signaling pathways. Our findings are in agreement with the findings of Asahara et al. [18] who used another experimental setting, the rabbit model of balloon angioplasty.

It is well known that mechanical injury induces a wave of endothelial proliferation [20]. The time course was described by Schwartz and Benditt [21] who found no apparent proliferation until 20-24 h after balloon injury, but a peak proliferation at $48 \mathrm{~h}$ and a decreased proliferation rate at $96 \mathrm{~h}$. Moreover, an infusion of VEGF for 3 min immediately after endothelial denudation by balloon injury increased endothelial cell proliferation in the vascular wall [22].

A recent publication by Gennaro et al. [23] describing a study in old rabbits demonstrated that an adenoviral strategy with a vector encoding for $\mathrm{VEGF}_{165}$ can signifi- cantly elevate VEGF expression in injured arteries, accelerate $\mathrm{rET}$ and reduce neointimal formation. Moreover, a single topical application of VEGF reduced intimal hyperplasia and improved endothelial function in a rabbit vein graft model [24].

\section{Effects of VEGF on Microvascular Remodeling}

The healing process of the vessel after trauma is predominantly dependent on the existence and connection of extracellular matrix proteins, such as collagens. Several collagens are necessary for the composition of the vascular wall and form up to $80 \%$ of the dry weight of blood vessels [25]. The vessels mainly contain collagen type I and III [26]. Collagen type I is mainly identified in the adventitia and collagen type III preferentially in the intima and media [27]. Collagen IV and laminin can be detected in basement membranes which are present in all epithelial and endothelial linings and also in muscles and nerves. Immunohistochemistry can visualize collagen IV and laminin in the subendothelial basement membrane and that surrounding vascular SMCs [28]. This subendothelial basement membrane is necessary for the rET process of denuded vessels. Friedrich et al. [28] detected collagen type III and IV in experimental microsurgical anastomoses of rat arteries. Collagen IV was distributed in a net-like pattern in the media, collagen type III was detected in the adventitia. We detected a few positive collagen type III fibers in normal vehicle-treated vessels. VEGF clearly increased the amount of collagen type III in the adventitia of a normal vessel. In vessels with anastomosis an impressing increase of collagen type I is detectable in the media and adventitia. Until today, no data exist concerning the impact of VEGF on the expression of extracellular matrix proteins in vascular wound healing. Here, we demonstrate for the first time that VEGF significantly increases several collagen types as well as fibronectin. In contrast to untreated anastomosed vessels, VEGF-treated vessels exerted a clear accumulation of collagen type III in the media and adventitia. A recent paper showed that VEGF induced fibronectin secretion by cultured human airway SMCs [7]. Our group has shown in a recent study [29] that inhibition of VEGF activity by PTK787/ZK222584, a specific blocker of both VEGF receptor tyrosine kinases, inhibits the growth of a poorly differentiated thyroid cancer when using a nude mouse xenograft tumor model. Extracellular matrix proteins are upregulated in different tumor xenografts, whereas tyrosine kinase inhibition significantly reduced the abundance of these extracellular matrix proteins in the interstitial space as well as the perivascular areas [29]. The 
mechanisms for these data are still unclear, but an increase in programmed cell death of the tumor cells is discussed.

Furthermore, we detected an increase of TGF- $\beta_{1}$ in VEGF-treated animals immediately at day 10 after reanastomosis. These data suggest that VEGF may play a role in stimulating vascular remodeling after vascular injury, either alone or through interactions with other growth factors that stimulate the expression of extracellular matrix proteins. TGF- $\beta_{1}$ is also increased in the vascular wall in response to balloon injury [30].

A very interesting and new finding is the early upregulation of osteopontin after vascular injury. Osteopontin is further elevated in VEGF-treated rats indicating a specific role of this cell adhesion molecule in wound healing and early vascular remodeling. Osteopontin is known to be a mediator of growth and the cytokine/extracellular matrix protein osteopontin (Eta-1) is an important component of cellular immunity and inflammation. It also acts as a survival, cell-adhesive, and chemotactic factor for endothelial cells [31]. Isoda et al. [32] used a transgenic mouse model overexpressing the OPN gene and demonstrated that OPN induces both medial thickening without injury and neointimal formation after injury suggesting a certain role in the development of vascular remodeling after angioplasty in vivo.

The reestablishment of an endothelial monolayer is important in cases of reconstructive vascular surgery and vascular trauma. Our data of the present study are of significant clinical relevance. The endothelium plays a critical role in thrombogenicity and vascular tone and delayed endothelial healing after injury, such as after surgical procedures (transplantation, replantation, reconstructive surgery).

Furthermore, osteopontin appears to play a key role in the vascular remodeling process following microsurgery. Here, we present novel data on the role of osteopontin in the process of remodeling.

In summary, our study demonstrates that local VEGF supplementation represents a very useful method to accelerate rET and vascular healing after microvascular injury and reanastomosis within the vascular wall of the rat femoral artery.

\section{References}

1 Bates DO, Harper SJ: Regulation of vascular permeability by vascular endothelial growth factors. Vasc Pharmacol 2003;39:225-237.

2 Ferrara N: VEGF: An update on biological and therapeutic aspects. Curr Opin Biotechnol 2000;11:617-624.

3 Ferrara N, Gerber HP, LeCouter J: The biology of VEGF and its receptors. Nat Med 2003; 9:669-976.

4 Ferrara N: Role of vascular endothelial growth factor in regulation of physiological angiogenesis. Am J Physiol Cell Physiol 2001;280: C1358-C1366.

5 Roberts WG, Palade GE: Increased microvascular permeability and endothelial fenestration induced by vascular endothelial growth factor. J Cell Sci 1995; 108:2369-2379.

6 Wang W, Dentler WL, Borchardt RT: VEGF increases BMEC monolayer permeability by affecting occluding expression and tight junction assembly. Am J Physiol Heart Circ Physiol 2001;280:434-440.

7 Kazi AS, Lotfi S, Goncharova EA, et al: Vascular endothelial growth factor-induced secretion of fibronectin is ERK dependent. Am J Physiol Lung Cell Mol Physiol 2004;286: L539-L545.

8 Grimm D, Cameron D, Griese DP, et al: Differential effects of growth hormone on cardiomyocyte and extracellular matrix protein remodeling following experimental myocardial infarction. Cardiovasc Res 1998;40:297-306.
9 Shakibaei M, John T, De Souza P, et al: Signal transduction by betal integrin receptors in human chondrocytes in vitro: Collaboration with the insulin-like growth factor-I receptor. Biochem J 1999;342:615-623.

10 Junqueira LCU, Cossermelli W, Brentani R Differential staining of collagens type I, II and III by Sirius red and polarization microscopy. Arch Histol Jpn 1978;41:267-274.

11 Grimm D, Huber M, Jabusch HC, et al: Extracellular matrix proteins in cardiac fibroblasts derived from rat hearts with chronic pressure overload: Effects of beta-receptor blockade. $\mathrm{J}$ Mol Cell Cardiol 2001;33:487-501.

12 Leung DW, Cachianes G, Kuang WJ, et al: Vascular endothelial growth factor is a secreted angiogenic mitogen. Science 1989;246:13061309.

13 Keck PJ, Hauser SD, Krivi G, et al: Vascular permeability factor, an endothelial cell mitogen related to PDGF. Science 1989;246:13091312.

14 Infanger M, Schmidt O, Kossmehl P, et al: Vascular endothelial growth factor serum level is strongly enhanced after burn injury and correlated with local and general tissue edema. Burns 2004;30:305-311.
15 Monacci WT, Merrill MJ, Oldfield EH: Expression of vascular permeability factor/vascular endothelial growth factor in normal rat tissues. Am J Physiol 1993;264:C995-C1002.

16 Jakeman LB, Winer J, Bennett GL, et al: Binding sites for vascular endothelial growth factor are localized on endothelial cells in adult rat tissues. J Clin Invest 1992;89:244-253.

17 Hamdan AD, Aiello LP, Misare BD, et al: Vascular endothelial growth factor expression in canine peripheral vein bypass grafts. J Vasc Surg 1997;26:79-86.

18 Asahara T, Chen D, Tsurumi Y, et al: Accelerated restitution of endothelial integrity and endothelium-dependent function after phVEGF165 gene transfer. Circulation 1996; 94:3291-3302.

19 Lindner V, Reidy MA: Expression of VEGF receptors in arteries after endothelial injury and lack of increased endothelial regrowth in response to VEGF. Arterioscler Thromb Vasc Biol 1996;16:1399-1405.

20 Couper LL, Bryant SR, Eldrup-Jorgensen $\mathrm{J}$, et al: Vascular endothelial growth factor increases the mitogenic response to fibroblast growth factor-2 in vascular smooth muscle cells in vivo via expression of fms-like tyrosine kinase-1. Circ Res 1997;81:932-939.

21 Schwartz SM, Benditt EP: Cell replication in the aortic endothelium: A new method for study of the problem. Lab Invest 1973;28:699_ 707. 
22 Burke PA, Lehmann-Bruinsma K, Powell JS: Vascular endothelial growth factor causes endothelial proliferation after vascular injury. Biochem Biophys Res Commun 1995;207: 348-354.

23 Gennaro G, Menard C, Michaud SE, et al: Agedependent impairment of reendothelialization after arterial injury: Role of vascular endothelial growth factor. Circulation 2003;107:230233.

24 Luo Z, Asahara T, Tsurumi Y, et al: Reduction of vein graft intimal hyperplasia and preservation of endothelium-dependent relaxation by topical vascular endothelial growth factor. J Vasc Surg 1998;27:167-173.
25 Kucharcz E: The Collagens: Biochemistry and Pathophysiology. New York, Springer, 1992, p 10.

26 Kuhn K: The classical collagens: Types I, II and III; in Mayne R, Burgeson RE (eds): Structure and Function of Collagen Types. Orlando, Academic Press, 1987, pp 1-42.

27 Farquharson C, Robins SP: Immunolocalization of collagens type I and III in the arterial wall of the rat. Histochem J 1989;21:172178.

28 Friedrich RE, Bartel-Friedrich S, Plambeck K: The presence of collagen III and IV in experimental microsurgical anastomoses of arteries. J Oral Maxillofac Surg 2000;58:1125-1128.

29 Schoenberger J, Grimm D, Kossmehl P, et al: Effects of PTK787/ZK222584, a tyrosine kinase inhibitor, on the growth of a poorly differentiated thyroid carcinoma: An animal study. Endocrinology 2004;145:1031-1038.
30 Miano JM, Vlasic N, Tota RR, et al: Smooth muscle cell immediate-early gene and growth factor activation follows vascular injury. A putative in vivo mechanism for autocrine growth. Arterioscler Thromb Vasc Biol 1993;13:211219.

31 Leali D, Dell'Era P, Stabile H, et al: Osteopontin (Eta-1) and fibroblast growth factor- 2 crosstalk in angiogenesis. J Immunol 2003; 171: 1085-1093.

32 Isoda K, Nishikawa K, Kamezawa Y, et al: Osteopontin plays an important role in the development of medial thickening and neointimal formation. Circ Res 2002;91:77-82. 